\title{
CAUSA SUI ET SUBSTANTIALITÉ LA RÉFORME DE LA NOTION DE SUBSTANCE, DE DESCARTES À SPINOZA ${ }^{1 *}$
}

Laurence Renault ${ }^{* *}$

\begin{abstract}
RESUMO
Oriundo de um curso proferido em 2012 na Unicamp e na UFU, o presente artigo tem duas ambições principais. Trata-se, por um lado, de estabelecer que a renovação da doutrina cartesiana da substância nos Princípios da Filosofia (I, 51), que vê o aparecimento de um novo sentido da substância, próprio a Deus, ao lado do sentido tradicional daquilo que existe em si, procede da doutrina de um Deus causa sui, elaborada nas Primeiras e Quartas respostas à objeções. Estabelecê-lo, uma vez que Descartes não evoca o Deus causa sui nos Princípios, isto é, não somente afirmá-lo, assim como o fizeram P. Aubenque e, depois, J.-M. Beyssade, mas demonstrá-lo. Trata-se, em segundo lugar, de mostrar como essa reinterpretação da substância se prolonga em Espinosa, segundo o qual toda substância é causa de si; mas também se realiza totalmente no que Espinosa faz da causa sui o correlato necessário do em si, de sorte que já não se trata de justapor um novo sentido ao antigo, mas de reformar o antigo, de introduzir uma dimensão de auto-causalidade na substancialidade, enquanto necessariamente requerido pela existência em si.
\end{abstract}

Palavras-chave: Descartes. Espinosa. Causa sui. Substância.

\section{RÉSUMÉ}

Le présent article, issu d'un cours donné en 2012 à l'Unicamp et à l'UFU, a deux ambitions principales. Il s 'agit, d'une part, d'établir que le renouvellement de la doctrine cartésienne de la substance dans les Principes de la philosophie (I, 51), qui voit l'apparition d'un nouveau sens de la substance, propre à Dieu, à côté du sens traditionnel de ce qui existe en soi, procède de la doctrine d'un Dieu causa sui, mise en œuvre dans les Premières et Quatrièmes réponses aux objections. L'établir,

1 *Le présent article est issu d'un cours donné en 2012 à l'invitation des Professeurs Eneias Forlin et Alexandre Guimaraes Tadeu de Soares à l'Unicamp puis à l'UFU. Je les remercie très vivement pour cette invitation et pour leur chaleureux accueil.

** Professora de Filosofia da Universidade de Paris-Sorbonne.E-mail: laurence.renault@, paris-sorbonne.fr 
puisque Descartes n'évoque pas le Dieu causa sui dans les Principes, c'est-à-dire non pas seulement l'affirmer, ainsi que l'ont fait P. Aubenque puis J.-M. Beyssade, mais le démontrer. Il s'agit, en second lieu, de montrer comment cette réinterprétation de la substance se prolonge chez Spinoza, selon lequel toute substance est cause de soi, ; mais, aussi, s'y parachève en ce que Spinoza fait de la causa sui le corrélat nécessaire de l'en soi, de sorte qu'il ne s'agit plus de juxtaposer un nouveau sens à l'ancien, mais bien de réformer l'ancien, d'introduire une dimension d'auto-causalité dans la substantialité, en tant que nécessairement requise par l'existence en soi.

Mots-clés: Descartes. Spinoza. Causa sui. Substance.

La notion de causa sui, notion forgée par Descartes - non pas au sens où il en serait l'inventeur, mais au sens où il est celui qui tente d'en établir la consistance et la cohérence ${ }^{2}$ - induit une refonte du concept de substance. Certes, l'émergence de la notion de cause de soi, en 1641, dans les Réponses aux premières objections faites aux Méditations Métaphysiques et son approfondissement, la même année, dans les Réponses aux quatrièmes objections, ne se font pas en lien avec la notion de substance. C'est à propos de la nature divine, de son rôle de cause première, et sans qu'il soit question de son statut de substance, que la thèse d'un Dieu causa sui apparaît dans les écrits de Descartes. Cependant, les remaniements de la doctrine cartésienne de la substance qu'on observe en 1644 dans le petit traité de la substantialité des articles 51-53 de la première partie des Principes de la philosophie semblent liés à la notion de causa sui. Ils consistent principalement dans l'apparition d'une différence, qui n'existait pas auparavant dans les écrits de Descartes, entre la notion de substance telle qu'on doit l'entendre lorsqu'il s'agit de la substance divine et la notion de substance créée, à laquelle correspond le sens traditionnel de ce qui existe per se, jusque là le seul en usage dans la pensée cartésienne. Or, la nouvelle signification de

2 Cf. MARION, J.-L. « La causa sui, Responsiones I et IV », in Questions cartésiennes II, Paris : PUF, 2002, p. 143 - 182, notamment p. 145 - 154. Une première version ce cet article est parue dans le volume Descartes, Objecter et répondre, BEYSSADE, J.-M.; MARION, J.-L. (Éd.). Paris : PUF, 1994. Nous ne nous engagerons pas ici dans l'analyse des difficultés de la notion cartésienne de causa sui, ni dans la détermination du type de causalité qu'elle recouvre (efficiente ? formelle ?), et ceci qu'il s'agisse de Descartes ou de Spinoza. Nous renvoyons pour ces questions à l'étude de J.-L. Marion que nous venons de citer, à l'ouvrage de CARRAUD, V. Causa sive ratio Paris, PUF, 2002. Nous nous intéresserons seulement à la question de savoir comment, avec Descartes, puis Spinoza, la substantialité acquiert une dimension d'auto-causalité, quelle qu'elle soit. 
la substantialité, celle qui est propre à Dieu, semble directement en lien avec la doctrine d'un Dieu causa sui. Et comme elle s'accompagne de l'affirmation selon laquelle Dieu seul est substance au sens propre du terme, c'est la notion de substance elle-même - et non seulement celle de substance divine - qui semble ainsi réinterprétée à la lumière de la doctrine d'un Dieu causa sui. Certes, la notion de cause de soi n'est pas explicitement présente dans ce passage des Principes, pas plus que dans le reste de l'ouvrage. Pour étayer une telle lecture ${ }^{3}$, il faut donc en premier lieu établir que le sens de la notion de substance propre à Dieu selon ce texte renvoie implicitement à la doctrine d'un Dieu causa sui. C'est à cette condition seulement qu' il est possible d'affirmer que c'est la notion de cause de soi qui infléchit l'analyse cartésienne de la notion de substance.

Si tel est bien le cas, alors, d'une part, on pourra dire que la substantialité en Dieu renvoie désormais pour Descartes au fait qu'il est cause de soi, et que l'aséité positive constitue le sens propre de l'indépendance dans l'existence qui caractérise la substance, alors que le sens traditionnel, selon lequel une substance est ce qui existe en soi ou per se, et non pas in alio, à la différence de l'accident, ne correspond plus qu'au type d'indépendance dans l'existence dont les substances créées sont capables relativement aux autres créatures et non pas à la subsistence au sens propre et absolu du terme.

D'autre part, on pourra affirmer que ce renouvellement de la notion de substance liée à l'émergence de la notion de cause de soi dans la pensée de Descartes se poursuit avec Spinoza, qui, dans l'Ethique, fait de la causa sui le caractère de toute substance. Ainsi la démonstration de la proposition VII de la première partie affirme-t-elle qu' « une substance ne peut être produite par autre chose : elle sera donc cause de soi $»^{4}$. Ce n'est pas le propre de la substance divine d'être cause de soi, en ce sens que c'est une caractéristique

3 Une telle lecture, dans sa ligne générale, ne nous est pas propre. Le premier à l'avoir mise en œuvre est Pierre Aubenque dans un article paru en 1992 : «La transformation cartésienne du concept aristotélicien de substance », dans le volume Le style de la pensée. En hommage à Jacques Brunschwig, Paris, Les belles lettres, p. 490-501, et repris dans Pierre Aubenque, Problèmes aristotéliciens, I, Philosophie théorique, Paris, Vrin 2009, p. 341350. Jean-Marie Beyssade a également proposé une lecture du traité de la substantialité de Principes I, 51-53 à partir de la causa sui dans « La théorie cartésienne de la substance. Equivocité ou analogie ?», in Revue internationale de philosophie 1996/1, p. 51-72, texte repris dans ses Etudes sur Descartes, Paris, Le Seuil, 2001, p. 217-244. Nous partageons l'optique générale de ces deux études, mais pas toujours le détail de leurs analyses.

${ }^{4}$ Ethique I, VII, dem : « substantia non potest produci ab alio ; erit itaque causa sui ». 
de la substance qui n'est pas liée à l'absolue infinité de Dieu, mais à la substantialité elle-même, ce que ne doit pas masquer la thèse spinoziste selon laquelle la seule substance existante est la substance divine : une substance infinie en son genre serait aussi nécessairement cause de soi. C'est ainsi, là encore, la notion de substance elle-même qui est réinterprétée et pas seulement celle de substance divine.

Or, ce qui se joue dans cette thèse spinoziste selon laquelle toute substance est cause de soi, et ce qu'apporte en propre la doctrine de Spinoza par rapport à celle de Descartes, c'est l'articulation, dans la notion de substance, de l'en soi et de la cause de soi. En effet, la caractérisation traditionnelle de la substance ne disparaît nullement chez Spinoza, et elle n'est pas non plus déclassée, puisque Spinoza n'admet pas d'équivocité de la notion de substance. Toute substance est donc substance au sens de ce qui existe en soi et de ce qui est cause de soi. Les deux caractéristiques distinguées par Descartes et définissant deux significations distinctes de la substance sont au contraire corrélées l'une à l'autre par Spinoza. La notion de causa sui apparaît ainsi comme la fine pointe de l'explicitation de la notion d'être en soi : ce qui est en soi est nécessairement cause de soi. Chez Spinoza, les nouvelles élaborations relatives au concept de substance induisent donc un approfondissement de la notion traditionnelle de substance elle-même, en ce sens que c'est l'en soi qui conduit à la causa sui. Les deux formes d'indépendance dans l'existence distinguées par Descartes sont désormais réciproques.

\section{L'évolution de la notion de substance dans les écrits de Descartes}

Dans l'article 51 de la première partie des Principes de la philosophie, Descartes explicite la notion de substance en ces termes: « lorsque nous concevons la substance, nous concevons seulement une chose qui existe en telle façon qu'elle n'a besoin d'aucune autre chose pour exister $\rangle^{5}$. Or, cela ne convient qu'à la substance divine, écrit Descartes. Cela n'exclut pas pourtant qu'il y ait d'autres substances, mais elles ne le sont pas au même sens, en effet, « quant à toutes les autres substances, nous percevons qu'elles

5 AT VIII-1, 24 : «per substantiam nihil aliud intelligere possumus, quam rem quae ita existit, ut nulla alia re indigeat ad existendum ». 
ne peuvent exister autrement que par le moyen du concours de Dieu ${ }^{6}$. Le nom de substance n'est donc pas univoque ${ }^{7}$. La version française du texte ajoute deux précisions : d'une part, la définition de la substance, dans sa littéralité ${ }^{8}$, convient aux deux types de substance, mais pas au même sens. Ce n'est donc pas simplement le nom de substance qui est équivoque, c'est la définition elle-même. D'autre part, Descartes précise en quel sens entendre cette définition lorsqu'il s'agit des substances créées. Descartes propose donc deux caractérisations de la substance, celle qui convient à Dieu et celle qui convient aux choses créées, mais sous couvert d'une unique définition qui va ainsi s'expliciter en deux sens différents. C'est précisément ici que se joue le glissement de sens relatif à la substantialité : la même définition de la substance (ce qui n'a besoin que de soi-même pour exister) peut s'entendre soit au sens traditionnel de ce qui existe en soi $^{9}$, soit en un sens nouveau (n'avoir besoin du concours d'aucune autre chose pour exister), qui devient le sens propre du terme.

Seul Dieu n'a besoin que de soi-même pour exister, car « il n'y a aucune chose créée qui puisse exister un seul moment sans être soutenue et conservée par sa puissance $\rangle^{10}$. Les substances créées ne sont pas des substances «à proprement parler $\rangle^{11}$, parce que leur existence suppose à chaque instant le concours de Dieu, qui les conserve. Sans ce soutien constant dans l'être, elles retourneraient au néant. On ne peut donc pas dire à leur propos qu'elles n'auraient besoin d'aucune autre chose pour exister, puisque leur existence

6 AT VIII-1, 24 : « alia vero omnes, non nisi ope concursus Dei existere posse percipimus ».

7 Sur ce point et son rapport avec la tradition, cf. MARION, J.-L. Sur la théologie blanche de Descartes, Paris : PUF 1981, I, 1 §7.

8 La traduction de la définition la transforme légèrement, sans qu'il soit possible d'y lire une modification de la thèse énoncée : là où le latin disait que la substance n'a besoin « d'aucune autre chose » pour exister, le texte français de Picot énonce que la substance est ce qui n'a besoin « que de soi-même » pour exister (AT IX-2, 47).

9 P. Aubenque écrit à propos de cette définition: Descartes « paraît reprendre une définition d'inspiration aristotélicienne, lorsqu'il énonce « ce que c'est que la substance » au $§ 51$ du livre I des Principes de la philosophie « lorsque nous concevons la substance, nous concevons seulement une chose qui existe en telle façon qu'elle n'a besoin que de soimême pour exister ». Au sens strict, ce texte ne dit rien de plus que ce qu'Aristote désigne sous la dénomination de « séparé » ou de " par soi » : la substance est séparée (choriston) à la différence des autres prédicaments qui ne sont pas « sans elle » $(Z, 1028 \mathrm{a} 27-34)$ ».

${ }^{10}$ AT IX-2, 47.

${ }^{11}$ AT IX-2, 47. 
dépend à tout moment de la puissance de Dieu.

Descartes évoque ici la doctrine de la création continuée, ou conservation, héritée de la tradition médiévale latine et de la scolastique tardive. Il avait déjà repris ce thème à son compte dans la troisième Méditation, puis dans les Premières réponses. S'interrogeant, dans la troisième Méditation, sur la question de savoir si lui-même pourrait être au cas où Dieu n'existerait pas, il avait indiqué qu' " une substance, pour être conservée dans tous les moments qu'elle dure, a besoin du même pouvoir et de la même action qui serait nécessaire pour la produire et la créer tout de nouveau, si elle n'était point encore $»^{12}$. Cette précision n'avait cependant alors aucune incidence sur la notion de substance, elle ne conduisait pas à affirmer que la substance créée ne pouvait être substance au sens propre du terme. Au contraire, la caractérisation de l'ego comme substance dans ce passage, semblait avoir pour fonction de souligner que cette dépendance relativement à la conservation ne compromettait pas la substantialité, et que les deux questions, celle de la subsistence, et celle de la dépendance à l'égard d'une action conservatrice éaient à distinguer. Dans l'ensemble de la troisième Méditation, la notion de substance conserve d'ailleurs son sens traditionnel, hérité d'Aristote, de ce qui est en soi, distingué de l'être en un autre, et cela qu'il s'agisse de la substance créée ou de Dieu. Descartes y définit en effet la substance par l'être en soi : « une substance, ou bien une chose qui est capable d'exister par soi $»^{13}$. Qui plus est, cette définition semble commune à Dieu et à la substance créée, non seulement parce que Descartes n'en propose pas d'autre ; mais encore parce que la notion de substance ne paraît pas recouvrir d'équivocité. En effet, s'interrogeant sur la cause de la réalité objective de l'idée qui nous représente Dieu comme une substance infinie, Descartes précise que seule la notion d'infini, et non celle de substance, requiert comme cause une autre réalité que l'être formel de l'ego : « encore que l'idée de la substance soit en moi, de cela même que je suis une substance, je n'aurais pas néanmoins l'idée d'une substance

\footnotetext{
${ }^{12}$ AT IX-1, 39. C'est la traduction française qui fait intervenir la notion de substance, le latin utilise le terme de " res », qui en est un équivalent dans le vocabulaire philosophique ; cf. AT VII, 49 : « perspicuum enim est $<\ldots>$ eadem plane vi et actione opus esse ad rem quamlibet singulis momentis quibus durat conservandam, qua opus esset ad eandem de novo creandam, si nondum existeret ».

${ }^{13}$ AT VII, 44 : res « quae per se apta est existere ».
} 
infinie, moi qui suis un être fini, si elle n'avait été mise en moi par quelque substance qui fût véritablement infinie $»^{14}$. La question de la conservation des créatures par la puissance divine n'ouvre donc pas, dans la troisième Méditation, sur le renouvellement de la notion de substance, contrairement à ce qu'il en sera dans l'article 51 de Principes $\mathrm{I}^{15}$.

Dans la lettre d'août 1641 à l'Hyperaspistes ${ }^{16}$ où se rencontrent aussi les thèmes de la substantialité des créatures et de la création continuée, le lien entre les deux questions semble déjà différent : « il ne faut point douter que si Dieu retirait une fois son concours, toutes les choses qu'il a créées retourneraient aussitôt dans le néant, parce que avant qu'elles fussent créées, et qu'il leur prêtât son concours, elles n'étaient qu'un néant : mais cela n'empêche pas qu'elles ne doivent être appelées des substances, parce que quand on dit de la substance créée qu'elle subsiste par elle-même, on n'entend pas par là exclure le concours de Dieu, duquel elle a besoin pour subsister, mais seulement, on veut dire qu'elle est telle qu'elle peut exister sans le secours

${ }^{14}$ AT VII, 45 : « quamvis substantiae quidem idea in me sit ex hoc ipso quod sim substantia, non tamen idcirco esset idea substantiae infinitae, cum sim finitus, nisi ab aliqua substantia, quae revera esset infinita, procederet ». J.-M. Beyssade, dans l'article cité supra propose une lecture, ainsi qu'une traduction, différentes de ce passage, mais à la lumière d'un écrit bien plus tardif de Descartes, la lettre à Clerselier du 23 avril 1649, (Etudes sur Descartes p. 229-230), ce qui ne nous semble précisément pas respecter l'évolution de la doctrine cartésienne dont nous faisons état.

${ }^{15}$ D'une telle univocité de la notion de substance témoignera encore l'Abrégé géométrique des Secondes réponses, qui définit ainsi la substance : « omnis res cui inest immediate, ut in subjecto, sive per quam existit aliquid quod percipimus, hoc est aliqua proprietas, sive qualitas, sive attributum, cujus realis idea in nobis est, vocatur Substantia. » définition V, AT VII, 161. Cette définition, qui se situe dans la lignée de l'hypokeimenon aristotélicien, est suivie de la définition de la substance pensante, de celle de la substance corporelle, et de celle de la substance divine. La notion de substance parait ainsi s'entendre univoquement, et en son sens traditionnel. Sur ce qui sépare, déjà, cette définition de la définition aristotélicienne de l'ousia comme hypokeimenon, voir P. Aubenque, article cité, Problèmes aristotéliciens, philosophie théorique, p. 346-347. Contrairement à J.-M. Beyssade (ibid. p. 234-235), nous ne lisons pas dans ce passage de dimension causale dans le rapport entre la substance et ses attributs.

${ }^{16}$ Lorsqu'il écrit cette lettre,Descartes a achevé la rédaction des six premières séries de réponses (en juillet 1641 pour les sixièmes). Ce texte, bien qu'écrit avant la parution effective des Méditations métaphysiques et des six premières séries d'objections et de réponses (en novembre 1641) est donc postérieur à l'élaboration de la notion de causa sui dans les premières et quatrièmes réponses. 
d'aucune autre chose créée, ce qui ne se peut dire de même des modes qui accompagnent les choses, comme sont la figure ou le nombre $\gg^{17}$. Ici apparaît la notion de « substance créée ", et, semble-t-il, l'idée que la notion de subsistence pourrait s'entendre au sens où elle exclurait tout concours, à la différence de la substantialité des créatures.

Dans l'article 51 de la première partie des Principes, l'action conservatrice de Dieu à l'égard des créatures intervient désormais explicitement dans la question de la substantialité, puisqu'elle implique que les substances créées ne peuvent pas être des substances au sens propre du terme. Ayant besoin du secours ordinaire de Dieu pour être maintenues dans l'existence, elles ont besoin d'autre chose qu'elles-mêmes pour exister, et ne vérifient donc pas pleinement, absolument, proprement, la définition de la substance. En quel sens la définition de la substance leur convient-elle? Au même sens que dans la lettre à l'Hyperaspistes : " pource qu'entre les choses créées quelques-unes sont de telle nature qu'elles ne peuvent exister sans quelques autres, nous les distinguons d'avec celles qui n'ont besoin que du concours ordinaire de Dieu, en nommant celles-ci des substances, et celles-là des qualités ou des attributs de ces substances $\gg{ }^{18}$. Le concours divin exclut qu'il y ait parmi les créatures des substances, au sens propre, c'est-à-dire absolu, du terme. Si certaines créatures peuvent être appelées des substances, c'est en un sens relatif au champ du créé (la substance creée est l'entité créée qui n'a pas besoin d'être soutenue dans l'être par d'autres créatures), et qui consiste dans la notion traditionnelle de subsistence, en tant qu'opposée à l'inhérence. La caractérisation traditionnelle de la substance, héritée d'Aristote, comme ce qui existe en soi, au titre de sujet dernier, ne correspond plus qu'à une acception impropre et relative de la substantialité. Telle est l'évolution de la doctrine cartésienne de la substance. Est-elle à mettre en rapport avec la doctrine d'un Dieu causa sui? Il reste à l'établir.

\footnotetext{
${ }^{17}$ «Nec dubium est, si Deus cessaret a suo concursu, quin statim omnia quae creavit in nihilum essent abitura, quia, antequam creata essent et ipsis concursum suum praeberet, nihil erant. Nec ideo minus vocari debent substantiae, quia, cum dicimus de substantia creata, quod per se subsistat, non ideo excludimus concursus divinum, quo indiget ad subsistendum ; sed tantummodo significamus illam esse talem rem, ut absque omni alia creata esse possit, quod idem de modis rerum ut de figura vel numero dici non potest ", AT III, 429.

${ }^{18}$ AT IX-2, 47. Cette précision est un ajout de la version française.
} 


\section{Conservation et causa sui selon Descartes}

Selon les Principes de la philosophie, Dieu est l'unique substance au sens propre du terme, en ce que, seul, il n'a besoin que de soi-même pour exister. Il reste cependant à déterminer en quel sens il faut entendre cette caractéristique propre à Dieu, puisque Descartes ne le précise pas. Que signifie que Dieu n'ait besoin d'aucun secours extérieur pour exister? Deux interprétations sont possibles, qui nous ramènent à l'alternative ouverte par Caterus dans les Premières objections : « je pense donc je suis, voire même, je suis l'esprit même et la pensée ; or, cette pensée et cet esprit, ou il est par soi-même, ou par autrui ; si par autrui, celui-là enfin par qui estil ? S'il est par soi, donc il est Dieu ; car ce qui est par soi se sera aisément donné toutes choses.

Ce mot par soi est pris en deux façons. En la première, il est pris positivement, à savoir par soi-même comme par une cause, et ainsi, ce qui serait par soi et se donnerait l'être à soi-même, si par un choix prévu et prémédité il se donnait ce qu'il voudrait, sans doute qu'il se donnerait toutes choses, et partant il serait Dieu. En la seconde, ce mot Par soi est pris négativement et est la même chose que de soi-même ou non par autrui, et de cette façon, si je m'en souviens, il est pris de tout le monde $»^{19}$.

Lorsque l'on dit qu'une entité existe par soi, cela peut s'entendre au sens de ce qui n'a pas besoin de cause, de ce qui est sans cause, ou bien au sens de ce qui n'a pas besoin de cause différente de soi-même, mais existe par soi-même comme par une cause. C'est-à-dire que cela peut s'entendre en un sens négatif ou bien en un sens positif. Par cette distinction, Caterus entend contrer l'argument de Descartes, selon lequel si l'ego existait par soi, il serait Dieu. En effet, une telle conséquence supposerait que l'ego soit

${ }^{19}$ AT VII, 94-95 : « Cogito, ergo sum, imo ipsa mens et cogitatio sum. Illa autem mens et cogitatio, aut a seipsa est, aut ab alio. Si hoc, istud porro a quo ? Si a se est, ergo Deus est : quod enim a se est, omnia sibi ipsi facile dederit. Rogo virum obsecroque, ut avidum Lectorem, et forte minus intelligentem, se non celet. Accipitur a se duplici modo. Primo, positive, nempe a seipso ut a causa ; atque ita, quod a se esset, sibique ipsi daret esse suum, si praevio delectu sibi daret quod vellet, haud dubie sibi omnia daret, atque Deus esset. Secundo, accipitur a se negative, ut sit idem quod seipso, aut non ab alio; atque hoc modo, quantum memini, ab omnibus accipitur ». Cette objection s'adresse à un passage de la troisième Méditation, où l'ego envisage l'hypothèse suivante : "si a me essem... » AT VII, 48. 
cause de soi-même. Or, cela n'est pas possible, car cette notion implique contradiction : la chose, comme cause, se précèderait elle-même, comme effet. On peut donc admettre l'hypothèse de Descartes selon laquelle l'ego existerait par soi, mais on ne peut pas l'invalider en en concluant que si tel était le cas, il se serait donné toutes les perfections qu'il conçoit dans l'idée de Dieu, puisque cela supposerait l'aséité entendue au sens positif, laquelle enveloppe contradiction. Tels sont le contexte et la portée de la distinction proposée par Caterus entre aséité positive et aséité négative. Comme on le sait, c'est à la faveur de cette objection de Caterus que Descartes va développer sa doctrine d'un Dieu causa sui : il n'est plus alors question de l'hypothèse selon laquelle l'ego serait par soi, il s'agit pour Descartes de défendre la notion d'aséité positive, dont il affirme qu'elle est requise par la notion de première cause : " la lumière naturelle nous dicte qu'il n'y a aucune chose de laquelle il ne soit loisible de demander pourquoi elle existe, ou dont on ne puisse rechercher la cause efficiente, ou bien, si elle n'en a point, demander pourquoi elle n'en a pas besoin. De sorte que, si je pensais qu'aucune chose ne peut en quelque façon être à l'égard de soi-même ce que la cause efficiente est à l'égard de son effet, tant s'en faut que de là je voulusse conclure qu'il y a une première cause, qu'au contraire de cellelà même qu'on appellerait première, je rechercherais derechef la cause et ainsi je ne viendrais jamais à une première $»^{20}$. La notion de cause première requiert que l'on rende raison du fait qu'une telle cause est première, c'està-dire qu'elle n'a pas elle-même besoin d'une cause différente pour exister, ce qui signifie qu'elle doit « en quelque façon être à l'égard de soi-même ce que la cause efficiente est à l'égard de son effet ». La seule manière d'éviter une régression à l'infini, ou bien un arrêt arbitraire dans la série des causes, fondé sur la seule « imperfection de l'esprit humain $»^{21}$ est donc d'admettre qu'une réalité peut être la cause de soi-même, causa sui : " Il peut y avoir quelque chose dans laquelle il y ait une puissance si grande et si inépuisable

${ }^{20}$ AT VII, 108-109: « Dicat autem profecto lumen naturae nullam rem existere, de qua non liceat petere cur existat, sive in ejus causam efficientem inquirere, aut, si non habet, cur illa non indigeat, postulare ; adeo ut, si putarem nulla rem idem quodammodo esse posse erga seipsam, quod est causa efficiens erga effectum, tantum abest ut inde concluderem aliquam esse causam primam, quin e contra ejus ipsius, quae vocaretur prima, causam rursus inquirerem, et ita nunquam ad ullam omnium primam devenirem » .

${ }^{21}$ AT VII, 110. 
qu'elle n'ait jamais eu besoin d'aucun secours pour exister, et qui n'en ait pas encore besoin maintenant pour être conservée, et ainsi qui soit en quelque façon la cause de soi-même, et je connais que Dieu est tel $»^{22}$.

Cette doctrine est-elle à l'arrière-plan du texte des Principes ? La définition selon laquelle « est substance ce qui n'a besoin que de soi-même pour exister », en son sens propre, qui ne convient qu'à Dieu, signifie-t-elle que Dieu n'a pas besoin de cause pour exister, ou bien qu'il est à lui-même sa propre cause ? Dieu est-il substance au sens où il serait sans cause, ou bien en ce qu'il serait causa sui ? ${ }^{23}$

${ }^{22}$ AT VII, 109 : « admitto aliquid esse posse, in quo sit tanta et tam inexhausta potentia, ut nullius unquam ope eguerit ut existeret, neque etiam nunc egeat ut conservetur, atque adeo sit quodammodo sui causa ; Deumque talem esse intelligo ».

${ }^{23} \mathrm{Ni}$ Aubenque, ni Beyssade, dans les articles cités plus haut, n'estiment nécessaire d'ouvrir cette question. P. Aubenque appuie sa lecture, semble-t-il, sur le fait que Dieu seul est substance, ce qui tient pour acquis que la conception d'un Dieu causa sui est maintenue dans les Principes : "Le pas qui fait de la substance la causa sui, étranger à Aristote, évité par Thomas d'Aquin et même encore par Suarez, est franchi — pour la première fois, semble-t-il —par Descartes. Dans le texte des Principes, il n'utilise pas l'expression causa sui, mais il remarque que, si la substance est définie comme il l'a fait, à savoir ce qui n'a besoin que de soi-même pour exister, seul Dieu peut être dit substance » Problèmes aristotéliciens I, p. 344. Selon J.-M. Beyssade « en définissant la substance comme ce qui existe sans avoir besoin de rien d'autre pour exister, on rejoint la notion positive de l'aséité, la cause de soi qui est une pensée neuve de la métaphysique cartésienne » Etudes sur Descartes, p. 232. Pour notre part, nous ne voyons pas en quoi cette définition ferait davantage signe vers l'aséité positive que vers l'aséité négative : un Dieu existant par soi au sens de « sans cause » serait également une chose « qui n'a besoin de rien d'autre pour exister ». Beyssade invoque, il est vrai dans la suite de son analyse, les caractéristiques des preuves de l'existence de Dieu dans les Principes : d'une part, le fait de placer au premier rang $(I, 14)$ la preuve a priori (mais ce type de preuve, déjà présent dans la cinquième Méditation, ainsi que dans la tradition, n'est pas nécessairement solidaire de la thèse d'un Dieu cause de soi); d'autre part, la manière dont Descartes conclut l'ensemble des preuves par les effets, dans l'article I, 21 : « nous connaissons aisément qu'il n'y a point de force en nous par laquelle nous puissions subsister, ou nous conserver un seul moment, et que celui qui a tant de puissance qu'il nous fait subsister hors de lui et qui nous conserve, doit se conserver soi-même, ou plutôt, n'a besoin d'être conservé par qui que ce soit, et enfin qu'il est Dieu » AT IX-2, 34. La thèse ici énoncée nous paraît beaucoup plus ambiguë que ce qu'en restitue Beyssade, qui commente ainsi : « il n'a aucun besoin d'aucune conservation par aucune autre chose puisqu'il est à lui seul sa propre cause ou raison d'exister » ibid. p. 233. Nous aurons l'occasion de revenir sur l'ambiguïté de cet article un peu plus loin. 
Ce qui nous semble constituer l'argument principal en faveur de la lecture selon laquelle la doctrine de la substance divine dans les Principes s'adosse à celle d'un Dieu causa sui, c'est le rôle, qui n'a guère été remarqué jusqu'ici, que joue le thème de la conservation dans les Premières réponses. L'argumentation en faveur de la doctrine d'un Dieu cause de soi présente en effet cette caractéristique frappante, dans ce texte, de se fonder principalement sur la question de la conservation. La notion d'un Dieu causa sui est rendue nécessaire, non seulement pour établir la primauté de la première cause (premier argument, qui établit la nécessité métaphysique de la notion de cause de soi) ; mais aussi pour rendre raison du fait que Dieu « est et ne cesse point d'être »: Dieu n'a besoin d'aucun secours pour exister, ni pour être conservé « parce que c'est lui-même qui en effet se conserve » (second argument, qui établit que Dieu est cause de soi). Il s'agit d'un argument qui peut surprendre en ce qu'il consiste à transposer à Dieu la notion de conservation, à l'aide de l'argumentation mise en œuvre dans la tradition pour établir que l'action conservatrice de Dieu est nécessaire au maintien dans l'être de toute créature : « tout de même que, bien que j'eusse été de toute éternité, et que par conséquent il n'y eût rien eu avant moi, néanmoins, parce que je vois que les parties du temps peuvent être séparées les unes d'avec les autres, et qu'ainsi, de ce que je suis maintenant, il ne s'ensuit pas que je doive être encore après, si, pour ainsi parler, je ne suis créé de nouveau à chaque moment par quelque cause, je ne ferais point de difficulté d'appeler efficiente la cause qui me créée continuellement en cette façon, c'est-à-dire qui me conserve. Ainsi, encore que Dieu ait toujours été, néanmoins, parce que c'est lui-même qui en effet se conserve, il semble qu'assez proprement il peut être appelé la cause de soi-même $»^{24}$. Certes, Descartes ne prétend cependant pas que Dieu existe dans le temps, ni qu'il ait besoin d'être « créé

${ }^{24}$ AT VII, 109 : nam quemadmodum, etiamsi fuissem an aeterno ac proinde nihil me prius extitisset, nihilominus, quia considero temporis partes a se mutuo sejungi posse, atque ita ex eo quod jam sim non sequi me mox futurum, nisi aliqua causa me quasi rursus efficiat singulis momentis, non dubitarem illam causam, quae me conservat, efficientem appellare : ita, etiamsi Deus nunquam non fuerit, quia tamen ille ipse est qui se revera conservat, videtur non nimis improprie dici posse sui causa ». La suite du texte reprend à plusieurs reprises l'expression suivante : " la cause pourquoi il est et ne cesse point d'être », AT VII $110,1.25$ et 1.28. Sur ce thème, dans la tradition, cf. par exemple F. Suarez, Disputationes metaphysicae XXI, I, 17 et II, 3, qui développe des arguments assez proches. 
de nouveau à chaque instant » ainsi qu'il en va de la créature. Cependant, il transpose bien à l'être divin la question de la conservation, réservée jusque là à la créature. Et il y répond en affirmant que Dieu se conserve dans l'être par sa propre puissance. La notion de conservation est ainsi au cœur de l'argumentation par laquelle Descartes défend la doctrine d'un Dieu cause de soi au moment où il l'énonce pour la première fois. C'est sous la forme de la question de la conservation que Descartes impose à Dieu la question de la cause.

L'intérêt d'un tel argument est manifeste. D'une part, il permet d'éviter que l'éternité de Dieu soit invoquée en faveur de l'aséité négative. Dieu ayant été de toute éternité, nulle cause ne serait requise pour rendre raison de son existence, puisqu'il n'a pas eu à venir à l'être : il serait ainsi par soi au sens de sans cause. C'est sans doute la raison pour laquelle Descartes introduit la question de la conservation divine à partir de l'hypothèse selon laquelle l'ego aurait été de toute éternité. Il y a ici un chiasme : on attribue, par hypothèse, l'éternité à l'ego et on s'autorise de cette hypothèse pour transposer à Dieu la question de la conservation, en tant qu'elle se poserait même à propos d'un esprit fini éternel. Descartes remarque en effet que l'éternité attribuée par hypothèse à l'ego n'empêche pas que sa persévérance dans l'existence requiert une cause par l'action de laquelle il est conservé dans l'existence (en tant qu'il demeure soumis aux conditions d'une existence temporelle, divisible en instants discontinus, lors même qu'on admet par hypothèse qu'il est de toute éternité, ce en quoi réside l'une des difficultés de l'argument). Il s'agit ici d'insinuer que la question de la conservation ne se pose pas seulement à propos des entités qui sont venues à l'être à un moment donné, mais à propos de toute entité, y compris éternelle, pour autant qu'il faut expliquer pourquoi elle ne cesse point d'être. Ce procédé argumentatif a ainsi pour fonction de déconnecter la notion de conservation de celle de création, autrement dit, de contester l'équivalence entre conservation et création continuée ${ }^{25}$.

D'autre part, l'intérêt de cette démarche argumentative reposant sur la

${ }^{25}$ Et ceci à l'encontre de ce que Descartes lui-même écrivait dans la troisième Méditation: « ut conservationem sola ratione a creatione differe, sit etiam unum ex is quae lumine naturali manifesta sunt » AT VII, 49 ; « la lumière naturelle nous fait voir clairement que la conservation et la création ne diffèrent qu'au regard de notre façon de penser », AT IX-2, 39. 
notion de conservation est qu'elle permet d'éviter la difficulté soulevée par Caterus, après bien d'autres auteurs de la tradition, selon laquelle la notion de cause de soi serait contradictoire en ce qu'elle impliquerait que la chose se précède elle-même. Il ne s'agit pas, avec la causa sui, de la cause en vertu de laquelle Dieu serait venu à l'être, car il n'y a jamais eu de commencement de l'existence divine. Il s'agit de la cause de l'existence actuelle de Dieu, ou plus exactement de la cause qui fait que cette existence ne cesse pas. Or, la cause conservatrice n'est pas antérieure à son effet, qu'elle maintient dans l'être. La conservation suppose que la chose existe déjà, la cause conservatrice n'est donc pas antérieure à son effet, pas même logiquement. Descartes l'a lui-même souligné dans les Premières réponses, à propos de la seconde preuve par les effets de la troisième Méditation: " j'ai mieux aimé appuyer mon raisonnement sur l'existence de moi-même, laquelle ne dépend d'aucune suite de causes, et qui m'est si connue que rien ne le peut être davantage ; et m'interrogeant sur cela moi-même, je n'ai pas tant cherché par quelle cause j'ai autrefois été produit, que j'ai cherché quelle est la cause qui à présent me conserve, afin de me délivrer par ce moyen de toute suite et succession de causes $»^{26}$. La question de la cause, appliquée à l'être divin, est donc celle de la conservation éternelle de Dieu dans l'être, laquelle réfère à sa propre puissance s'exerçant actuellement, sans tomber sous le coup de la contradiction selon laquelle la chose qui serait causa sui devrait se précéder elle-même dans l'être pour être sa propre cause ${ }^{27}$. Seule une entité éternelle peut ainsi être cause de soi sans que cette notion enveloppe contradiction.

Descartes n'insiste pas davantage sur ce thème de la conservation dans les Quatrièmes réponses. Arnauld a contesté l'usage qu'en faisaient les Premières réponses par deux objections. a) La question de la conservation

${ }^{26}$ AT IX-1, 85 ; AT VII, 107 : « de me non tam quaesivi a qua causa olim essem productus, quam a qua tempore praesenti conserver, ut ita me ab omni causarum successione liberarem ».

${ }^{27}$ Arnauld a parfaitement aperçu l'intérêt de cet argument : «on ne peut pas concevoir que Dieu soit par soi positivement, comme s'il s'était lui-même premièrement produit, car il aurait été auparavant que d'être ; mais seulement (comme notre auteur le déclare en plusieurs lieux), parce qu'en effet il se conserve » AT IX-1, 165 ; AT VII ? 221-212 : « Deus a se positive cogitari non potest, quasi seipsum primo produxerit : fuisset enim antequam esset ; sed tantum (ut saepius daclarat Author) quia se revera conservat ». Il conteste cependant qu'on puisse dissocier la conservation de la création. 
suppose la temporalité, elle ne peut s'appliquer à ce qui est éternel ${ }^{28}$; b) la conservation renvoie à la création, c'est-à-dire à la continuelle reproduction de la chose ${ }^{29}$. Descartes ne renonce pourtant pas à appliquer la notion de conservation à l'être divin, dans sa réponse à Arnauld. Mais il le fait d'une manière assez discrète : " et il ne se trouvera point d'endroit, où $\mathrm{j}$ 'aie dit que Dieu se conserve pas une influence positive, ainsi que les choses créées sont conservées par lui, mais bien seulement ai-je dit que l'immensité de sa puissance ou de son essence, qui est la cause pourquoi il n'a pas besoin de conservateur, est une chose positive $»^{30}$. Il semble estimer qu'il a déjà,

${ }^{28}$ AT IX-1, 164 «Dans l'idée d'un être infini, l'infinité de sa durée y est aussi contenue, c'est-à-dire qu'elle n'est point renfermée dans aucunes limites, et partant qu'elle est indivisible, permanente et subsistante tout à la fois, et dans laquelle on ne peut sans erreur et qu'improprement, à cause de l'imperfection de notre esprit, concevoir de passé, ni d'avenir. D'où il est manifeste qu'on ne peut concevoir qu'un être infini existe, quand ce ne serait qu'un moment, qu'on ne conçoive en même temps qu'il a toujours été et qu'il sera éternellement (ce que notre auteur même dit en quelque endroit); et partant, que c'est une chose superflue de demander pourquoi il persévère dans l'être. Voire même, comme l'enseigne saint Augustin $\langle\ldots>$ en Dieu, il n'y point de passé ni de futur, mais un continuel présent ; ce qui fait voir clairement qu'on ne peut sans absurdité demander pourquoi Dieu persévère dans l'être, vu que cette question enveloppe manifestement le devant et l'après, le passé et le futur, qui doivent être bannis de l'idée d'infini »; AT VII, 211 : « In idea enim entis infiniti continetur, quod ejus duratio sit etiam infinita, scilicet nullis clausa limitibus, ac proinde indivisibilis, permanens, tota simul, et in qua non nisi per errorem et intellectus nostri imperfectionem concipi possit prius et posterius. Unde manifeste sequitur concipi non posse Ens infinitum vel per momentum existere, quin simul concipiatur et semper extitisse, et in aeternum existentiam habiturum (quod Author ipse alicubi docet), ut proinde vanum sit quaerere cur in se perseveret. Imo, ut frequenter docet Augustinus $<\ldots>$ in Deo nullum est vel fuisse vel futurum esse, sed semper esse ; ut hinc evidentius appareat, non nisi cum absurditate quaeri posse cur Deus in esse perseveret, cum haec quaestio manifeste involvat prius et posterius, praeteritum et futurum, quae $a b$ Entis infiniti notione excludi debent $»$.

${ }^{29}$ AT IX-1, 165 : «La conservation ne convient pas mieux à l'être infini que la première production. Car qu'est-ce, je vous prie, que la conservation, sinon une continuelle reproduction d'une chose ? d'où il arrive que toute conservation suppose une première production »; AT VII, 212 : « at in ens infinitum conservatio non magis cadit quam prima productio. Quid enim, quaeso, conservatio, nisi continua quaedam rei reproductio ? Unde conservatio omnis supponit primam productionem $»$.

${ }^{30}$ AT IX-1, 183 ; AT VII, 236-237 : « nec ullibi dixi Deum se conservare per positivum aliquem influxum, ut res creatae ab ipso conservantur, sed tantum immensitatiem potentiae sive essentiae, propter quam conservatore non indiget, esse rem positivam ». 
par avance en quelque sorte, répondu aux critiques d'Arnauld : « je puis facilement admettre tout ce que M. Arnauld apporte pour prouver que Dieu n'est pas la cause efficiente de soi-même et qu'il ne se conserve pas par aucune influence positive, ou bien par une continuelle reproduction de soimême, qui est tout ce que l'on peut inférer de ses raisons $\gg{ }^{31}$. Dieu se conserve par sa propre puissance, non pas au sens où il se produirait de nouveau (la conservation n'est pas une réitération de la première production, dans le cas d'un être éternel), ni au sens où l'on supposerait qu'il existe dans le temps.

On peut, certes, estimer que cet argument ne résout pas tous les problèmes liés à la notion de causa sui, et qu'il est lui-même problématique. Toujours est-il que c'est celui que Descartes utilise dans les Premières réponses, qu'il l'estime au dessus des critiques d'Arnauld dans les Quatrièmes réponses, et qu'il l'invoque encore dans les Principes de la philosophie. En effet, ce thème est présent à l'article 21 de la première partie, quoique d'une manière qui atteste de la prudence de Descartes à son égard. Il écrit en effet : « nous connaissons aisément qu'il n'y a point de force en nous par laquelle nous puissions subsister ou nous conserver un seul moment, et que celui qui a tant de puissance qu'il nous fait subsister hors de lui et qui nous conserve doit se conserver soi-même, ou plutôt n'a besoin d'être conservé par qui que ce soit, et enfin qu'il est Dieu $»^{32}$. S'il avait renoncé à concevoir l'être éternel de Dieu du point de vue de la conservation, pourrait-il encore écrire, même pour revenir immédiatement en deçà de cette affirmation, que Dieu se conserve lui-même?

C'est à la lumière du rôle que joue la notion de conservation dans la question de la causa sui, et de sa présence dans l'article 21 que l'on peut établir que Descartes suggère dans l'article 51 de la première partie des Principes, que Dieu est substance au sens propre du terme, à la différence des substances créées, en ce qu'il se conserve lui-même dans l'existence,

${ }^{31}$ AT IX-1, 183 ; AT VII, 237 : « itaque possum ultro admottere quaecunque a Viro Cl. asseruntur ad probandum Deum non esse causam efficientem sui ipsius, neque se conservare per ullum influxum positivum, sive per sui reproductionem continuam ; quod solum ex ejus rationibus efficitur. »

${ }^{32}$ AT IX-2, 34 ; AT VIII-1, 13 : « facile enim intelligimus nullam vim esse in nobis, per quam nos ipsos conservemus ; illumque in quo tanta est vis, ut nos a se diversos conservet, tanto magis etiam se ipsum conservare, vel potius nulla ullius conservatione indigere, ac denique Deum esse ». 
c'est-à-dire en ce qu'il est causa sui. Dieu est donc substance, dans les Principes de la philosophie, au sens où il se soutient lui-même dans l'existence, c'est-à-dire au sens où il existe et persévère dans l'existence par sa propre puissance, à la différence des substances créées qui ont besoin du secours divin pour persévérer dans l'être. La substantialité, au sens propre du terme, ne renvoie donc plus à la subjectité, au fait d'être sujet, c'est-à-dire de ne pas avoir besoin d'autre chose comme sujet, mais revêt une dimension causale. Elle désigne la manière dont une entité se soutient elle-même, causalement, dans l'existence, et subsiste au sens où elle persévère dans l'être par sa propre puissance.

\section{Spinoza: l'univocité retrouvée.}

L'élaboration spinoziste de la notion de substance, dans l'Ethique, se situe dans la lignée de la réinterprétation cartésienne que nous venons d'analyser. En effet, selon la doctrine de l'Ethique, toute substance est cause de soi. La substantialité est donc corrélative du fait d'être cause de soi. En cela, Spinoza s'inspire de Descartes, et de son interprétation de la notion de substance divine dans les Principes. Mais il va aussi plus loin que Descartes dans la réforme de la notion de substance. En effet, selon Descartes, seule la substance divine est cause de soi, la substantialité des créatures doit s'entendre en un sens différent, puisque toute créature a besoin du secours ordinaire de Dieu pour être et pour se conserver dans l'être. Certes, c'est la notion même de substance qui est engagée, en ce que c'est en Dieu seul qu'on l'entend au sens propre du terme. Mais, ce sens propre ne peut convenir qu'à l'être divin. Pour Spinoza, en revanche, toute substance est cause de soi, une substance ne saurait être que cause de soi. Il en résulte qu'il n'y a plus chez Spinoza deux manières d'entendre la substantialité mais une seule.

Pour bien comprendre ce que signifie ce retour à un sens un, univoque, de la substance, chez Spinoza, et de quelle manière se prolonge chez lui la réforme de la notion de substance, il importe de donner plusieurs précisions. $1^{\circ}$ ) Tout d'abord, la thèse selon laquelle toute substance est cause de soi ne signifie pas que Spinoza abandonne le sens traditionnel de la substance, pour ne conserver que la nouvelle caractérisation de la notion de substance, héritée de Descartes. En effet, toute la réflexion spinoziste sur la substance, dans l'Ethique, s'adosse à la caractérisation de la substance par la subsistence, 
opposée à l'inhérence. Les définitions données au début de la première partie en attestent : " par substance, j'entends ce qui est en soi et se conçoit par soi $»^{33}$; «par mode, j'entends les affections d'une substance, autrement dit, ce qui est en autre chose et se conçoit aussi par cette autre chose $»^{34}$. C'est à partir de cette caractérisation traditionnelle de la substance que Spinoza va établir qu'une substance est toujours cause de soi. Autrement dit, Spino$\mathrm{za}$, en soutenant que toute substance est cause de soi, ne fait pas un choix entre les deux sens distingués par Descartes pour ne conserver que le sens propre. La signification traditionnelle de la substance est toujours présente chez Spinoza, elle est même au fondement de l'élaboration du traité de la substantialité que constituent les propositions 1 à 8 de la première partie de l'Ethique. Mais, s'il n'y a plus qu'une seule signification de la notion de substance, c'est qu'il articule ces deux caractères (l'en soi et la causa sui) au sein de l'unique notion de substance qu'il admet. Ce n'est donc pas parce que l'un disparaîtrait au profit de l'autre, mais parce qu'une connexion nécessaire est établie par Spinoza entre les deux sens distingués par Descartes.

$2^{\circ}$ ) C'est en cela que Spinoza à la fois prolonge la réforme cartésienne de la substance (puisqu'il reprend le sens nouveau forgé par Descartes) mais aussi qu'il la parachève (parce qu'il ne se contente pas de juxtaposer le nouveau sens et l'ancien, mais qu'il intègre à la notion traditionnelle de substance la caractéristique d'être cause de soi). Pour Spinoza, toute substance est cause de soi, et cela, dans la mesure même où elle est en soi, comme on va le voir. C'est parce qu'elle est un être en soi et non pas un être en un autre que la substance est nécessairement cause de soi. Le sens forgé par Descartes devient ainsi la vérité du sens traditionnel, la causa sui apparaît comme le réquisit jusque là inaperçu de l'être en soi.

Enfin, $3^{\circ}$ ) cette thèse ne signifie pas que Spinoza accorderait aux étants finis, d'être cause de soi. Elle signifie au contraire qu'aucun étant fini ne peut avoir le statut de substance. Davantage, aucune entité causée ne peut être substance, tout ce qui est produit par l'action d'une cause a raison de mode. Ce qui est $a b$ alio est toujours aussi in alio, c'est là le revers de la

${ }^{33}$ Définition III : « Per substantiam intelligo id, quod in se est, et per se concipitur ». Nous citons l'Ethique dans l'édition bilingue de B. Pautrat, Paris, éditions du Seuil, Points essais $n^{\circ} 380,2010$.

${ }^{34}$ Définition V : «Per modum intelligo substantiae affctiones, sive id, quod in alio est, per quod etiam concipitur ». 
connexion entre en soi et cause de soi $^{35}$, que Descartes évitait précisément par sa thèse de l'équivocité du terme de substance. Mais cela ne signifie pas pour autant que la causa sui serait une prérogative de la substance divine, comme c'est le cas chez Descartes. En effet, dans le traité de la substance qui occupe les huit premières propositions de l'Ethique, on ne raisonne pas sur Dieu, mais sur les propriétés de la substance en général, telle qu'elle a été définie au début de la première partie, comme ce qui est en soi et se conçoit par soi. L'absolue infinité qui caractérise la substance divine ne joue aucun rôle dans la démonstration de la corrélation entre la subsistence, au sens traditionnel, et la cause de soi. Que la substance soit infinie en son genre ou absolument infinie, elle est nécessairement cause de soi. Qu'il n'existe et ne peut existerqu'une seule substance, absolument infinie, qui est Dieu, ne change rien à la portée de cette analyse.

\section{La démonstration de la corrélation entre être en soi et causa sui}

Dans la définition de la substance posée au début de la première partie de l'Ethique entrent deux caractéristiques : la substance est ce qui est en soi et se conçoit par soi. Spinoza précise immédiatement cette seconde caractéristique : « c'est-à-dire, ce dont le concept n'a pas besoin du concept d'une autre chose d'où il faille le former $»^{36}$. C'est sur cette caractéristique de l'être en soi de se concevoir par soi que Spinoza va s'appuyer pour démontrer les propriétés de la substance, notamment celle d'être cause de soi.

D'où vient cette affirmation, suivant laquelle une substance non seulement est un être en soi, mais aussi se conçoit par soi ? Pourquoi est-elle corrélative de l'existence en soi de la substance? C'est Descartes qui a développé ce thème, et affirmé cette corrélation. Selon lui, on reconnaît une substance au fait que l'on peut concevoir clairement et distinctement la nature d'une chose séparément d'une autre. Par exemple, nous concevons clairement et distinctement la nature de la pensée sans faire intervenir quoi que ce soit qui relève de la matière, c'est-àdire de l'étendue : aucune détermination géométrique n'entre dans la conception

${ }^{35}$ Cela déborde la seule question des êtres finis et existants dans la durée, puisque Spinoza admet des modes infinis et éternels, qui sont précisément tels «par leur cause » (les modes infinis). L'infinité et l'éternité ne sont pas des prérogatives de la substance.

${ }^{36}$ La suite de la définition III précise : « hoc est, id, cujus conceptus non indiget conceptu alterius rei, a quo formari debeat ». 
claire et distincte que nous avons de la pensée. Nous pouvons donc concevoir la pensée sans faire intervenir le concept de l'étendue. De cette caractéristique de la pensée d'être concevable par soi, Descartes dérive l'affirmation selon laquelle la pensée n'a pas besoin de l'étendue pour exister, et constitue ainsi une substance différente de la substance étendue ${ }^{37}$. Cette caractéristique de la substance d'être conçue par soi est chez Descartes la transcription cognitive de l'indépendance dans l'être de la substance. L'indépendance de l'essence d'une chose, dans la conception claire et distincte que nous en formons, est pour Descartes le critère, dans notre connaissance, de ce qui est substance, de ce qui peut exister en soi.

Spinoza reprend cette caractéristique de la substance de se concevoir par soi. Mais c'est au prix de deux modifications décisives. $1^{\circ}$ ) Ce qui est en soi se conçoit par soi. Son concept se forme sans rien emprunter au concept d'une autre chose. C'est le critère de la substantialité, mais ce n'est plus le critère de la distinction substantielle : tout ce qui est concevable par soi est substance, mais à tout attribut concevable par soi ne correspond pas nécessairement une substance différente de celle que nous percevons par un autre attribut conçu par soi. $2^{\circ}$ ) Spinoza applique strictement cette caractéristique de la substance de se concevoir par soi, en ce qu'il en déduit qu'il ne saurait y avoir deux substances de même nature : « dans la nature des choses, il ne peut y avoir deux ou plusieurs substances de même nature ou attribut $\gg^{38}$. En effet, si tout substance se conçoit par soi, on ne peut pas admettre que deux substances aient quelque chose en commun. Cela signifierait que le concept de l'une envelopperait le concept de l'autre, et vice-versa. Elles ne seraient donc pas deux substances.

Cette caractéristique de la substance, héritée de Descartes, joue le rôle de moyen terme dans la démonstration de la propriété de toute substance d'être cause de soi. Spinoza s'appuie sur le fait que la substance est conçue par soi pour établir la connexion entre l'être en soi et la cause de soi. Cette démonstration a lieu dans les propositions 5 à 7 de Ethique I. C'est à partir de la thèse selon laquelle il ne peut pas y avoir deux substances de même nature

${ }^{37}$ Sur le rapport entre essence et substance chez Descartes, cf. AUBENQUE, P. « Sur l'ambivalence du concept aristotélicien de substance », Ontologie et dialogue. Hommage à Pierre Aubenque, CORDERO, N. (Éd.) Paris : Vrin 2000, p. 95 - 105, repris dans AUBENQUE, P. Problèmes aristotéliciens, Philosophie théorique, Paris : Vrin 2009. p. 197 - 209.

${ }^{38}$ «In rerum natura, non possunt dari duae, aut plures substantiae ejusdem naturae, sive attributi ». 
(prop. V) que Spinoza démontre qu'une substance ne peut pas être produite par une autre substance (prop VI). Il utilise pour cela la proposition 3: « des choses qui n'ont rien de commun entre elles, l'une ne peut être cause de l'autre $\gg{ }^{39}$. La démonstration de cette proposition repose elle-même sur deux axiomes énoncés au début de la première partie. L'axiome 5, selon lequel " les choses qui n'ont rien de commun entre elles ne peuvent non plus se comprendre l'une par l'autre, autrement dit, le concept de l'une n'enveloppe pas le concept de l'autre $»^{40}$. Et l'axiome $4:$ « la connaissance de l'effet dépend de la connaissance de la cause et l'enveloppe $»^{41}$. La dépendance dans laquelle l'effet est par rapport à sa cause a pour corrélat, sur le plan de la connaissance, que la connaissance de l'effet enveloppe celle de la cause. Le concept de l'effet doit envelopper celui de sa cause. Il s'ensuit qu'il ne peut pas y avoir de rapport de causalité entre deux substances, puisque chacune se conçoit par soi: « une substance ne peut pas être produite par une autre substance $\gg^{42}$ (prop. VI). La caractéristique des substances d'être conçues par soi, elle même corrélative de leur existence en soi, induit qu'une substance ne peut pas être cause d'une autre substance, dans la mesure où il ne saurait y avoir de rapport causal entre des choses qui sont différentes en nature.

La proposition VII tire la conséquence de ces démonstrations. Si une substance ne peut jamais être produite par autre chose, alors c'est qu'une substance existe en vertu de sa propre nature : «à la nature d'une substance appartient d'exister $»^{43}$. Ce qui signifie qu'une substance existe en tant qu'elle est cause de soi : « une substance ne peut être produite par autre chose; elle sera donc cause de soi ; c'est-à-dire son essence enveloppe nécessairement l'existence, autrement dit, à sa nature appartient d'exister $\rangle^{44}$.

Cette proposition et sa démonstration énoncent ainsi deux choses : l'aséité de la substance, et l'interprétation positive de cette aséité. De l'affirmation selon laquelle une substance ne peut être produite par autre chose, on passe

\footnotetext{
39 «Quae res nihil commune inter se habent, earum una alterius causa non esse potest».

40 "Quae nihil commune cum se invicem habent, etiam per se invicem intelligi non possunt, sive conceptus unius alterius conceptum non involvit ».

${ }^{41}$ «Effectus cognitio a cognitione causae ependet et eandem involvit»

${ }^{42}$ «Una substantia non potest produci ab alia substantia ».

43 " Ad naturam substantiae pertinet existere ».

44 «Substantia non potest produci ab alio; erit itaque causa sui, id est, ipsius essentia involvit necessario existentiam, sive ad ejus naturam pertinet existere ».
} 
en effet à l'affirmation selon laquelle elle est cause de soi. Autrement dit : si elle n'a pas de cause différente d'elle-même, c'est qu'elle est à elle-même sa propre cause. Ce que Spinoza explicite en précisant que son essence enveloppe l'existence. Cela renvoie à la définition de la cause de soi qui ouvre l'Ethique: " par cause de soi,j'entends ce dont l'essence enveloppe l'existence, autrement dit, ce dont la nature ne peut se concevoir qu'existante $»^{45}$. Une telle définition pouvait paraitre, étrangement, ramener la cause de soi à l'aséité négative de la tradition $^{46}$. Mais ce que veut dire Spinoza, c'est, tout au contraire, et comme le soutenait déjà Descartes, qu'il n'y a pas d'autre manière, pour une essence, d'envelopper l'existence, que d'être cause de soi, pas d'autre aséité que positive. En effet, la caractéristique de la substance d'être cause de soi est ensuite corrélée par Spinoza avec l'infinité. Spinoza démontre que toute substance est infinie, (c'est la proposition 8). Il produit pour cela deux démonstrations. Or, la seconde démonstration (dans le scolie I de la proposition 8) s'appuie sur la proposition 7 : « comme être fini est, en vérité, partiellement négation, et être infini affirmation absolue de l'existence d'une certaine nature, il suit donc de la seule proposition 7 que toute substance doit être infinie $»^{47}$. C'est dans ce scolie que Spinoza énonce l'équivalence entre le fait d'être cause de soi et l'affirmation absolue de l'existence d'une certaine nature. La cause de soi est donc bien à entendre comme aséité positive. L'essence qui enveloppe l'existence est telle parce qu'elle est une essence s'affirmant absolument dans l'existence. Toute essence de substance est donc puissance infinie ou absolue d'affirmation dans l'existence. Toute substance est donc bien cause de soi, au sens positif de l'aséité.

\section{Conclusion}

On retrouve ici un thème cartésien, celui qui consiste à expliciter la

${ }_{45}$ « Per causam sui intelligo id, cujus esssentia involvit existentiam, sive id, cujus natura non potest concipi, nisi existens ».

${ }^{46}$ Ce qu'avait relevé J.-L. Marion en ces termes : « cette implication $<\ldots>$ ne s'énonce aucunement en termes de cause (ni d'effet) et ne peut donc, du moins immédiatement, et avec l'autorité d'une définition, équivaloir à la causa sui », ibid. p. 146 Même remarque chez CARRAUD, V. Ibid., p. 297 et note 2.

${ }^{47} \mathrm{I}, 8$, scolie I, dem. : « Cum finitum esse revera sit ex parte negatio, et infinitum absoluta affirmatio existentiae alicujus naturae,sequitur ergo ex sola 7. prop. omnem substantiam debere esse infinitam $»$. 
causa sui à partir de la puissance inépuisable de la nature divine. C'est en vertu de son essence que Dieu existe, mais au sens où cette essence infinie s'accompagne, voire se confond avec l'essence divine, dans l'unité de ses perfections. Chez Spinoza, de même, l'essence infinie conçue par soi, l'essence d'une substance, est puissance d'affirmation absolue dans l'existence.

Cependant, chez Descartes, c'est la souveraine perfection de Dieu qui impose ainsi la causa sui, et la réinterprétation de la substance à partir de la cause de soi, dans laquelle l'aséité positive semble se substituer à l'existence en soi. Chez Spinoza, ce n'est pas la souveraine perfection de Dieu, mais simplement l'existence en soi de la substance qui impose la causa sui. La substance ne saurait être en soi si elle n'était cause de soi. L'indépendance au sens de la perséité serait compromise par toute dépendance causale, ce qui n'est pas le cas chez Descartes, où les deux questions ne se recouvrent pas: ainsi, la substance créée est en soi sans avoir à être cause de soi.

On pourrait, il est vrai, se poser la question suivante : pourquoi devrait-on interpréter positivement l'aséité de la substance comme causa sui ? Spinoza n'ouvre pas le débat, et semble au contraire se reposer sur un acquis de la tradition. Il considère apparemment que la cause de soi est une notion qui s'est imposée avant lui, et dont la cohérence et la consistance ont d'ores et déjà été établies. Qui plus est, cette question est plus simple à résoudre chez lui qu'elle ne l'est chez Descartes, parce qu'elle ne concerne pas d'emblée Dieu. En effet, à raisonner à partir de la notion de substance, ce n'est pas directement à Dieu qu'on impose la question de la cause, mais seulement à la substance, puis indirectement à Dieu en tant qu'il est substance. Or, personne ne soutiendrait qu'il faudrait s'abstenir de poser la question de la cause en vertu de laquelle une substance existe.

Cela étant, ce qui justifie l'aséité positive, c'est aussi l'exigence que toute substance soit elle-même la source de ses modes. On pourrait dire l'exigence de spontanéité de la substance, qui découle des analyses spinozistes. En effet, étant conçue par soi, toute substance doit être elle-même cause de ses modes, rien ne peut lui advenir de l'extérieur. Ainsi, la substance est en soi, conçue par soi et cause de soi et le mode est en un autre par lequel il est à la fois conçu et causé. La distinction en soi/en autre chose se redouble d'une dimension causale : ce qui est en soi est causa sui; ce qui est en autre chose est causé par autre chose. Mais c'est aussi la relation de la substance à ce qui existe en elle, à ses modes, qui acquiert une dimension causale, 
puisque c'est la substance elle-même qui est cause de ses modes. Le mode n'est pas simplement causé, il est causé par la substance dont il est un mode.

Or, cette spontanéité de la substance s'enracine précisément chez Spinoza dans l'interprétation de l'essence de substance comme puissance d'affirmation absolue dans l'existence. C'est en vertu de la même causalité que l'essence s'affirme absolument dans l'existence et déploie les modifications qui sont enveloppées en elle. Comme l'écrit Spinoza, à propos de la substance divine, mais cela vaudrait aussi bien d'une substance infinie en son genre : " au sens où Dieu est dit cause de soi, il faut aussi le dire cause de toute chose $»^{48}$. En effet, c'est en vertu de son essence qu'il est cause de soi et c'est également de la seule nécessité de son essence que suivent toutes les modifications qu'il produit. $\mathrm{Ce}$ point est confirmé par la proposition 34 et sa démonstration : « la puissance de Dieu est son essence même $»^{49}$. La démonstration indique en effet : « de la seule nécessité de l'essence de Dieu il suit qu'il est cause de soi et de toutes choses $\rangle^{50}$. Là encore, une telle démonstration n'est pas liée à l'absolue infinité de Dieu, c'est-à-dire à l'infinité de ses attributs, mais aux caractéristiques de chaque attribut, en tant qu'il est essence de substance. C'est donc aussi la nécessaire spontanéité de la substance qui requiert la causa sui.

Au terme de cette intégration progressive d'une dimension causale dans l'interprétation de la notion de substance, celle-ci se trouve considérablement transformée. Non seulement n'est en soi que ce qui est cause de soi, autrement dit, c'est en tant qu'elle s'affirme absolument dans l'existence que l'essence de la substance se confère à elle-même l'existence en soi. Mais encore, elle n'est plus substrat au sens d'un soubassement ontologique statique, à la manière de l'hypokeimenon aristotélicien, mais s'interprète désormais comme le dynamisme d'une puissance d'exister, et de porter à l'existence ses modifications. La substance soutient ses modes dans l'existence, non seulement au sens où ils ne peuvent exister qu'en elle, mais au sens où elle est la cause de leur existence.

Data de registro: $01 / 07 / 2015$

Data de aceite: 30/07/2015

\footnotetext{
${ }^{48}$ I, 25, scolie : « eo sensu quo Deus dicitur causa sui, etiam omnium rerum causa dicendus est ».

49 «Dei potentia est ipsa ipsius essentia »

${ }^{50}$ «Ex sola enim necessitate Dei essentiae sequitur, Deum esse causam sui et omnium rerum ».
} 